International Journal of Medical Sciences

ISSN 1449-1907 www.medsci.org 2006 3(2):28-28

(C)2006 Ivyspring International Publisher. All rights reserved

Editorial

\title{
Guest Editor's Editorial: Advances in Managing Hepatitis C Virus (HCV) Infection (A Special Issue)
}

\section{Ke-Qin Hu}

Division of Gastroenterology and Hepatology, University of California, Irvine Medical Center, CA 92868, USA

Corresponding address: Ke-Qin Hu, MD, Director of Hepatology Services and Associate Professor of Clinical Medicine, Divisions of GI and Transplantation, Univ. of California, Irvine Medical Center,101 The City Drive, Building 53, Suite 113, Orange, CA 92868, USA. Phone: 714-456-6745. Email: kqhu@uci.edu

Published: 2006.04 .01

\section{Editorial}

Chronic infection of hepatitis C virus (HCV) affects approximately 170 million people worldwide and 4-5 million people in the Unites States. Studies have projected a substantial burden from HCV disease and related complications, including liver failure and hepatocellular carcinoma (HCC), over the next 10 to 20 years. Since the identification of $\mathrm{HCV}$ in 1989, significant advances have been made in our understanding of HCV virology, diagnosis, natural history, and treatment of chronic HCV infection. The goal of this special issue of International Journal of Medical Sciences is to report and update our current knowledge in the selected areas.

The issue is started with a thorough review of the advances in HCV molecular virology by Drs. Brass, Moradpour, and Blum. Understanding HCV life cycle and the novel in vitro HCV replicating system will facilitate discovery of new anti-HCV drugs. Drs. Chevaliez and Pawlotsky describe the currently available serologic and molecular assays and their application in diagnosing HCV infection and monitoring HCV treatment. Understanding natural history of chronic HCV infection is essential for evidence-based management of HCV-infected patients. Drs. Sy and Jamal present a systematical overview on the epidemiology of chronic HCV infection, and Drs. Chen and Morgan provide a detailed update of our current understanding of natural history of HCV-related disease. Hepatic steatosis is a common presentation in HCV-infected individuals. Drs. Yoon and Hu review the incidence, pathogenesis, and clinical impact of steatosis on natural history of chronic HCV infection. Co-infection of hepatitis B virus (HBV) with HCV represents another challenge that is especially common in areas where HBV is prevalent. Drs. Liu and Hou give us an update on this important topic.

Perhaps, the most noteworthy advances with chronic HCV infection over the past decade have been the improved response rate to anti-HCV therapy. Drs. Huang and Hu review our practical approach to managing chronic hepatitis $\mathrm{C}$ from diagnosing and staging HCV disease to assessing candidacy, selecting, administering, and monitoring standard $\mathrm{HCV}$ treatments. It is well known that the sustained virological response (SVR) rate depends on a variety of virological and host factors, and the HCV treatment should be individualized. Management of chronic hepatitis $\mathrm{C}$ in special population is reviewed by Drs. Aulakh and Hoefs. HCV-related end-stage liver disease represents the leading cause of liver transplantation in the Unites States. Recurrent hepatitis $C$ has been associated with graft failure. Drs. Herrine and Navarro update pre-liver transplant treatment of patient with decompensated liver disease. Drs. Schiano and Martin review post-liver transplant treatment of recurrent hepatitis $C$.

I would like to thank all the contributors of this special issue for their time, experience, and insights to these important topics. Also, I wish that this special issue will be a valuable resource for your clinical practice.

\section{Conflict of interest}

The author has declared that no conflict of interest exists. 